\title{
Primary Research on Off-resonance Response of Underground Magnetic Resonance Sounding in Tunnel
}

\author{
Kerui Fan, Shucai Li, Bin Liu \\ Geotechnical and Structural Engineering Research \\ Center, Shandong University, Jinan, China
}

\author{
Xiu $\mathrm{Li}^{*}$ \\ Geology Engineering and Geomatics, Chang'an \\ University, Xi'an, China
}

\begin{abstract}
We present in this paper a forward modelling of the three dimensional off-resonance response of underground MRS used in advanced geological prediction of constructing tunnel. By considering and summarizing the off-resonance response in SNMR, we deduced the off-resonance response of underground MRS. Using three dimensional finite element method, a series of numerical examples are made to elaborate the feature of off-resonance response. We conclude that frequency offset and pulse duration determine the value of the offresonance response together. Under the excitation of standard pulse (almost $40 \mathrm{~ms}$ ), receiving voltage could not be reliable as long as frequency offset is larger than $5 \mathrm{~Hz}$; and this frequency offset could be smaller with longer duration of excitation pulse.
\end{abstract}

Keywords-MRS; off-resonance; tunnel

\section{INTRODUCTION}

Water and mud inrush are a predominantly geologic hazards that endangers the safety of men, especially in the construction of deep tunnel ${ }^{[1]}$. In this situation, advanced geological prediction is the essential and necessary to prospect the hazard body, instruct proper treatment and protect the safety of tunnel construction. Magnetic resonance sounding (MRS) is a new technology has been applied to investigate for quantitative analysis of ground water ${ }^{2 \sim 3]}$. The reliability and effectiveness of MRS inversion can be improved when combining with DC resistivity method or transient electromagnetic method (TEM), which are capable of exploring the distribution of subsurface conductivity in advance. Comparing with DC resistivity method, TEM and MRS are both electromagnetic induction method. The excitation of both methods can be realized by using transmitting coils with current pulse of specific waveform. The underground distribution of conductivity can be obtained via TEM survey, and MRS can give information of the existence, volume and porosity of the aquifer based on the advanced TEM survey ${ }^{[4 \sim 8]}$. Xiu et al. proposed a joint interpretation method that interprets TEM and MRS data together. The conductivity depth imaging (CDI) interpretation of TEM data can provide reasonable geo-electrical model, therefore the accuracy of the MRS inversion will be significantly improved. Thus, combining the advantage of both methods, joint interpretation and inversion of MRS and TEM may also provide a feasible solution to discriminate water from mud in prospected geological hazardous body, which is now a key issue in the research of advanced geological prediction for water and mud inrush in tunnel.
Though well practiced in surface detection of ground water (the SNMR), the underground application of MRS puzzles the researchers due to the limited excitation magnetic moment and complicated electromagnetic noise in tunnels. Besides, the MRS application is based on the crucial resonance condition i.e. the excitation frequency should be equal to the Larmor frequency. The violation against it leads to unreliable offresonance response. Consequently, the inversion may produce misinterpretation in terms of electrically conductive bodies or deeper aquifer occurrence ${ }^{[9]}$. Moreover, instrumental imperfections associated with the tuning of the energizing pulse may cause off-resonance effects or phase shifts. To explore the mechanism of off-resonance response in SNMR, researches on different aspects about it have been done by many researchers. Trushkin et al. studied the MRS signal excited and observed at different frequencies from both theoretical and practical viewpoints ${ }^{[10]}$. It is demonstrated that the characteristic frequency of NMR signal oscillations depending on detuning is inversely proportional to the exciting pulse duration. Legchenko and Valla and Girard et al. considered the off-resonance effect in signal detection and data processing ${ }^{[11]}$. Walbrecker et al. examined off-resonance effect in surface NMR based on the spin dynamics. Numerical and physical simulation are conducted to analyse the offresonance effect on single and double pulse SNMR. It is demonstrated that single pulse SNMR with standard 40mspluse produce reliable water contents for frequency offsets within $5 \mathrm{~Hz}$, corresponding to the condition of small to moderate pulse moments or deep aquifer occurrences. Whereas in the situation of strong pulse moments or the presence of shallow water, the resultant out-of-phase magnetization components must be considered for practically any frequency offset. Grombacher and Knight discussed the impact of off-resonance effect on water content estimation in the scenarios where magnetic susceptibility contrasts lead to a distribution of processional frequencies ${ }^{[11]}$.

So far, the off-resonance effect of underground MRS has not been widely focused by the researchers. Because of the limited working space and weak response amplitude of the underground MRS, the response may be easily overwhelmed in the noises even under a slight frequency offset. The study on the off-resonance response of the underground MRS is essential to conclude the response feature and give the constructive suggestion to the MRS application in underground engineering. 


\section{THREE-DimENSIONAL OFF-RESONANCE RESPONSE OF UNDERGROUND MRS}

In order to unify the expression of functions, $\mathbf{A}$ and $\hat{\mathbf{A}}$ are respectively used to represent a vector field and the unit vector along its direction. Scalar field, amplitude of a vector or matrix are represented in the form of $A$.

The principle of NMR is well known in physics which is energy level transition of proton. Its application in geophysics is based on the magnetic dipole moments of spins of hydrogen protons under the interaction of geomagnetic field and an artificially magnetic field at Larmor frequency. The hydrogen protons in water molecules absorb the energy and transit from the lower level to the upper level, as long as the excitation frequency $f_{\mathrm{T}}$ equals to Larmor frequency $f_{\mathrm{L}}$.

$$
f_{T}=f_{L}=\frac{\left|\boldsymbol{\omega}_{\mathbf{L}}\right|}{2 \pi}=\frac{\gamma_{p}\left|\mathbf{B}_{\mathbf{0}}\right|}{2 \pi}
$$

The $\omega_{\mathrm{L}}$ is the angular frequency corresponding to the Larmor frequency, and $\gamma_{p}$ is the gyromagnetic ratio for hydrogen protons. In the rotating frame, the tipping angle $\theta$ is determined according to ${ }^{[9]}$

$$
\theta=\gamma_{p}\left|\mathbf{B}_{\mathrm{T}}^{\perp+}\right| t
$$

where $t$ is the duration of the excitation pules, and $\mathbf{B}_{\mathrm{T}}^{\perp+}$ is the clockwise component of the exciting field, perpendicular to the earth field ${ }^{[12]}$.

$$
\mathbf{B}_{\mathrm{T}}^{\perp}=\mathbf{B}_{\mathrm{T}}-\left(\hat{\mathbf{B}}_{\mathbf{0}} \cdot \mathbf{B}_{\mathrm{T}}\right) \mathbf{B}_{0}=\mathbf{B}_{\mathrm{T}}^{\perp+}+\mathbf{B}_{\mathrm{T}}^{\perp-}
$$

The amplitude of $\theta$ tells the extent of excitation of hydrogen protons, per volume of water. However, as the excitation frequency deviates from the Larmor frequency, the frequency offset between $f_{\mathrm{T}}$ and $f_{\mathrm{L}}$ is given by

$$
\Delta f=\frac{\left|-\gamma_{p} \Delta \mathbf{B}_{0}\right|}{2 \pi},
$$

where

$$
\Delta \mathbf{B}_{\mathbf{0}}=\mathbf{B}_{\mathbf{0}}-\mathbf{B}_{\mathbf{0}}^{*}=-\frac{\boldsymbol{\omega}_{\mathbf{L}}-\boldsymbol{\omega}_{\mathrm{T}}}{\gamma_{p}}=-\frac{2 \pi \Delta f}{\gamma_{p}} \hat{\boldsymbol{\omega}} .
$$

The $\mathbf{B}_{\mathbf{0}}^{*}$ is the changed static field corresponding to $f_{\mathrm{T}}$ and $\hat{\boldsymbol{\omega}}$ is the unit vector along the direction of $\boldsymbol{\omega}$ The minus of the rightest item indicates that the direction of Larmor procession is opposite to the static field. And therefore, the effective tipping angle is determined according to

$$
\theta_{\text {eff }}(\mathbf{r}, t)=\gamma_{p}\left|\mathbf{B}_{\text {eff }}^{+}\right| t=\gamma_{p}\left(\sqrt{\left|\mathbf{B}_{\mathbf{T}}^{\perp+}\right|^{2}+\left|\frac{2 \pi \Delta \mathbf{f}}{\gamma_{p}}\right|^{2}}\right) t,
$$

where $\mathbf{B}_{\text {eff }}^{+}$is the clockwise component of the effective field $\mathbf{B}_{\text {eff }}$, which is given by ${ }^{\text {[9] }}$

$$
\mathbf{B}_{\text {eff }}=\mathbf{B}_{\mathrm{T}}^{\perp}-\left(\frac{2 \pi \Delta \mathbf{f}}{\gamma_{p}} \hat{\mathbf{B}}_{\mathbf{0}}^{*}\right) \mathbf{B}_{\mathbf{0}}^{*} .
$$

As consequence, the spin-magnetic-moment will not rotate along the axes- $x$ but the axes- $x^{*}$, the included angle between which is given by ${ }^{[9]}$

$$
\alpha=\tan ^{-1} \frac{2 \pi \Delta f}{\left|\gamma_{p} \mathbf{B}_{\mathrm{T}}^{\perp+}\right|} .
$$

With frequency offset, the value of kernel function is no longer real but complex. The kernel function of off-resonance response of underground MRS response is given as follow

$$
K_{3 D}^{*}=\frac{2 \pi f_{T} M_{0}^{*}}{I_{0}} B_{T}^{\perp^{*}}\left(\sin \left(\theta_{e f f}\right) \cos (\alpha)+i \sin ^{2}\left(\frac{\theta_{e f f}}{2}\right) \sin (2 \alpha)\right),
$$

where

$$
\begin{gathered}
\theta_{\text {eff }}=\sqrt{\left|\gamma \frac{B_{T}^{\perp^{*}}(\rho(\boldsymbol{r}), \boldsymbol{r})}{2}\right|^{2}+(2 \pi \Delta f)^{2}} \frac{q}{I_{0}}, \\
\alpha=\tan ^{-1} \frac{2 \pi \Delta f}{\left|\gamma \frac{B_{T}^{\perp^{*}}(\rho(\boldsymbol{r}), \boldsymbol{r})}{2}\right|} .
\end{gathered}
$$

The magnetic induction $B_{T}^{\perp^{*}}$ in (9) is the magnitude of excitation field perpendicular to current geomagnetic field $\mathbf{B}_{0}^{*}$. It can be calculated with known newly orientated inclination and declination of the geomagnetic field according to ${ }^{[14]}$

$$
B_{T}^{\perp^{*}}=R_{I} R_{D} R_{\alpha}^{T} R_{\beta}^{T} B_{T}
$$

where the $R_{I}$ and $R_{D}$ are the inclination and declination matrices of the geomagnetic field, the $R_{\alpha}^{T}$ and $R_{\beta}^{T}$ are the rotating matrices of the excitation coils.Finally, using volume integral the off-resonance response of underground MRS is given by

$$
E_{0}^{*}(q)=\int_{V} K_{3 D}^{*} \cdot n(\boldsymbol{r}) d v
$$

where $n(\boldsymbol{r})$ is the water content in a unit volume. At the consequence of complex kernel function, the value of the sounding curve $E_{0}{ }^{*}(q)$ is complex too. The receiving voltage is just the real part of $E_{0}{ }^{*}(q)$. And due to its pluralization, the phase of $E_{0}{ }^{*}(q)$ is also not equal to zero as well.

\section{THREE-DIMENSIONAL FORWARD MODELING OF UNDERGROUND MRS RESPONSE CONSIDERING THE OFF- RESONANCE EFFECT}

In order to verify the conclusion and summarize feature of off-resonance responses of the underground MRS, forward modelling of three-dimensional MRS response considering the off-resonance effect have been conducted using three- 
dimensional finite element method for different configurations. The background resistivity is assumed to be $700 \Omega \mathrm{m}$. The anomaly resistivity, water content and dimension of underground water bearing body are fixed as $10 \Omega \mathrm{m}, 50 \%$ and $10 \mathrm{~m} \times 10 \mathrm{~m} \times 5 \mathrm{~m}$ respectively. The Geomagnetic data used are $\mathrm{B} 0=52688.90 \mathrm{nT}, \mathrm{I}=54.8982 \mathrm{rads}$, and $\mathrm{D}=-6.1006$ rads (the geomagnetic data are available on the web site of www.ngdc.noaa.gov/seg/geomag/geomag.shtml).

The corresponding Larmor frequency is almost 2243.36 $\mathrm{Hz}$. The transmitting coil is square shaped with a side length of $6 \mathrm{~m}$. The water bearing body is ten-meters ahead of tunnel face. The excitation frequency varies from 2223.36 to 2263.36 $\mathrm{Hz}(\Delta f=-20 \sim+20 \mathrm{~Hz})$ in order to simulate the response affected by off-resonance effects. The model configuration is shown in Fig.1.

Firstly, off-resonance responses with different frequency offsets are simulated. The pulse durations of the excitation is $40 \mathrm{~ms}$. The pulse moments is given by varying the amplitude of the excitation current. As is shown in Fig. 2 (a) and Fig. 2 (b), the value of sounding curves is no longer real when $\mathrm{f} \neq$ 0. For the real part, shown in Fig. 2 (a), the amplitude decays with phase shifts in a sinusoid-related way in consequence of the increasing frequency offsets. Meanwhile, the waveform of the curves are distorted apparently, when the frequency offset is larger than $5 \mathrm{~Hz}$. The imaginary part of on-resonance response, shown in Fig.2 (b), equals to zero, as the consequence of (9). Unlike the real parts, the rest of them are rather intricate.

Secondly, off-resonance response under different pulse duration is simulated to illustrate the duration effect on it. Standard SNMR pulse with exactly 80 cycles of sinusoid wave is applied. Given different excitation frequencies, the pulse durations are differ from each other. Fig. 3 shows three simulations with frequency offsets of 0 and $\pm 20 \mathrm{~Hz}$. The difference in pulse duration contributes to these asymmetrical responses with the same value of $|\Delta f|$ according to (6) and (9).

Eventually, all the forward data, with excitation frequency from 2223.36 to $2263.36 \mathrm{~Hz}$, are synthesized in Fig. 4 (a) and Fig. 4 (b). Not only the 80-cycle pulses but also the 160 -cycle ones, $80 \mathrm{~ms}$ approximately, are used in the forward modellings. There are four features that can be observed and concluded from Fig. 4 and the above simulation. (i) When $\Delta f=0 \mathrm{~Hz}$, resonance condition is met, resulting in the maximal response

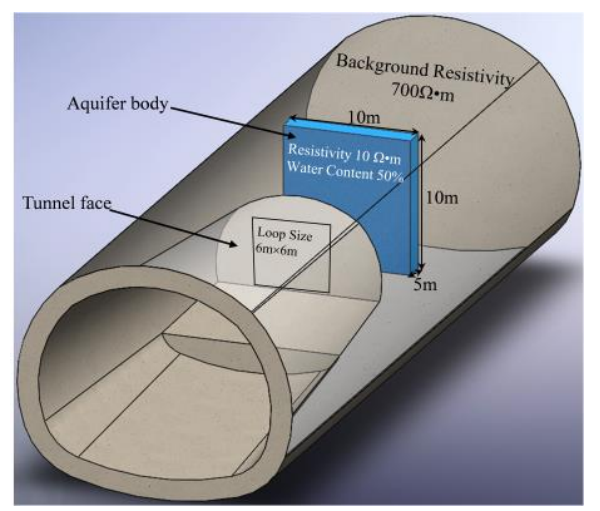

Fig.1. Model configuration.

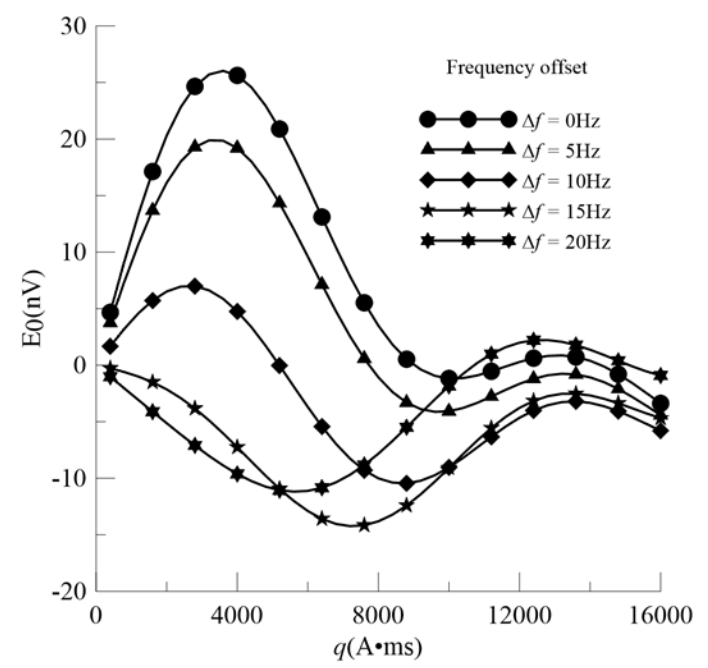

(a)

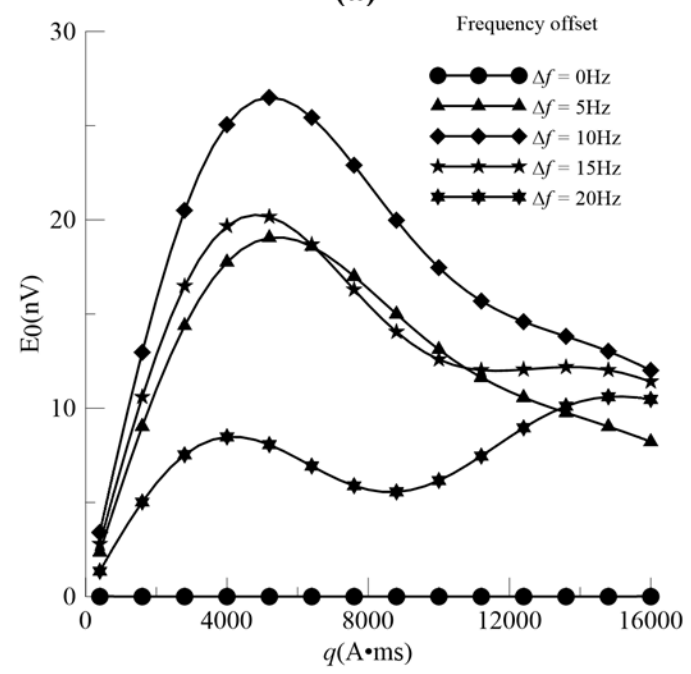

(b)

Fig.2. Off-resonance responses of the underground MRS in tunnel with different frequency offsets.

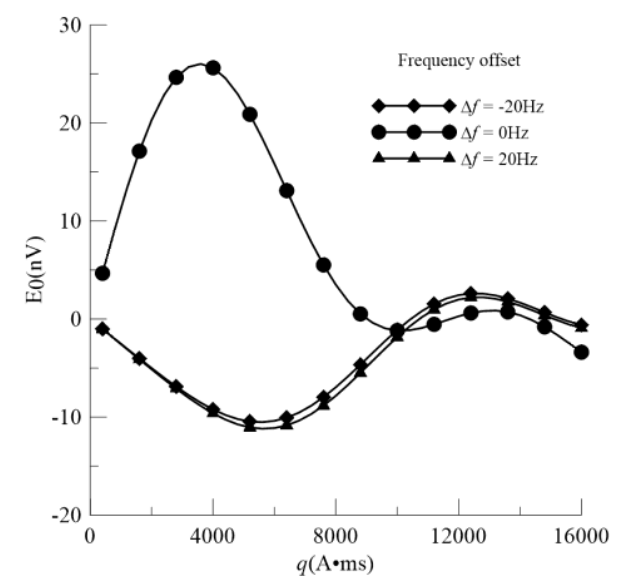

Fig.3. The asymmetry of the off-resonance response of the underground MRS in tunnel. 
(a)

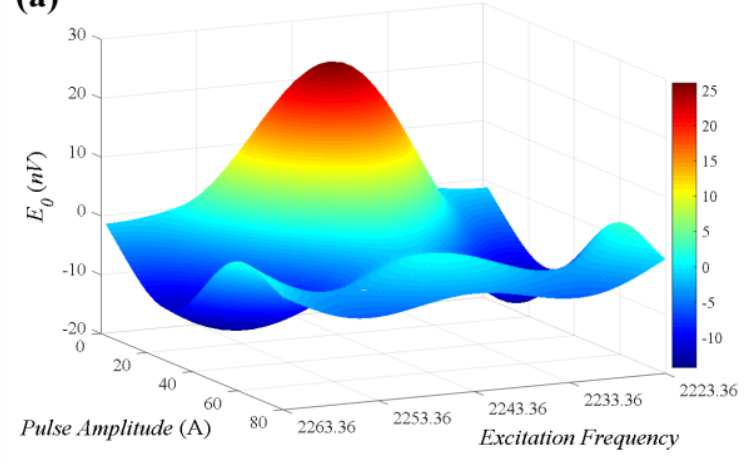

(b)

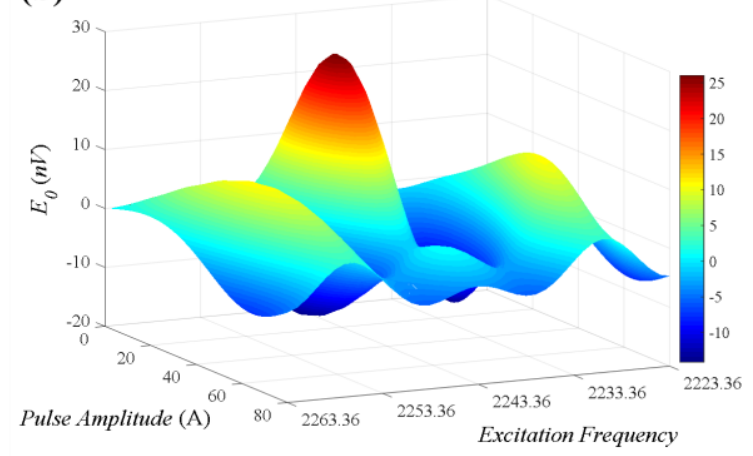

Fig.4. Synthetizations of the underground off-resonance responses with the frequency offsets ranging from $-20 \mathrm{~Hz}$ to $+20 \mathrm{~Hz}$.

amplitude shown in the middle of Fig. 4 (a) and Fig. 4 (b). (ii) When $\Delta f \neq 0 \mathrm{~Hz}$, the initial amplitudes decays according to (9) and (10). Under the excitation of pulses with same durations, the responses distribute on symmetry of Larmor frequency. (iii) The increase of $\Delta f$ contributes to the attenuation on the response amplitude in the form as the waves pass by. (iv) For a given frequency offset, the longer pulse duration contributes more violent off-resonance response, comparing Fig. 4 (a) and Fig. 4 (b). It looks the off-resonance response with larger frequency offset comes earlier in the presence of longer pulse duration. Considering the off-resonance effects, the frequency offsets in the applied excitation pulses should be suppressed as much as possible.

\section{CONCLUSION}

From the forward modelling of the off-resonance response in tunnel, we conclude that the off-resonance response is determined by frequency offset and the excitation pulse duration. Firstly, for aquifers with same depth, their response amplitudes decays with the increase of frequency offset $\Delta f$. secondly, given the same aquifer depth and frequency offset, the longer pulse duration leads to a more violent off-resonance effects. We suggest that the response voltage can't be reliable when frequency offset is larger than $5 \mathrm{~Hz}$. Unreliable sounding curves may lead to deeper location of the water bearing body, fallacious water content or unreal aquifer ahead of tunnel face. When applying the pulse with longer pulse duration, the offresonance effect for any practically frequency offset should be taken into account to avoid the excitation frequency offset in the application of the underground MRS. In the future works, the multi-frequency excitation and signal acquisition method and the excitation using frequency scanning should be focused in seek of the reliable excitation and the optimal response in the applications of underground MRS.

\section{REFERENCES}

[1] S. C. Li, B. Liu, H. F. Sun, L. C. Nie, S. H. Zhong, M. X. Su, et al., "State of art and trends of advanced geological prediction in tunnel construction," Chinese Journal of Rock Mechanics and Engineering, vol. 33(6), pp. 1090-113, June 2014.

[2] A. Legchenko, and P. Valla, "Processing of surface proton magnetic resonance signals using non-linear fitting," Journal of Applied Geophysics, vol. 39(2), pp. 77-83, February 1998.

[3] A. A. Behroozmand, K. Keating, and E.Auken, "A review of the principles and applications of the NMR technique for near-surface characterization," Surveys in Geophysics, vol. 36, pp. 27-85, July 2015.

[4] A. D. Parsekian, and D. Grombacher, "Uncertainty estimates for surface nuclear magnetic resonance water content and relaxation time profiles from bootstrap statistics," Journal of Applied Geophysics, vol. 119, pp. 61-70, September 2015.

[5] M. Hertrich, and U. Yaramanci, "Joint inversion of Surface Nuclear Magnetic Resonance and Vertical Electrical Sounding," Journal of Applied Geophysics, vol, 50, pp. 179-191, 2002.

[6] J. M. Vouillamoz, M. Descloitres, J. Bernard, P. Fourcassier, and L. Romagny, "Application of integrated magnetic resonance sounding and resistivity methods for borehole implementation. A case study in Cambodia," Journal of Applied Geophysics, vol. 50(1), pp. 67-81, 2002.

[7] A Legchenko, M. Ezersky, J. F. Girard, J. M. Baltassat, M. Boucher, C. Camerlynck, and A. Al-Zoubi, "Interpretation of magnetic resonance soundings in rocks with high electrical conductivity," Journal of Applied Geophysics, vol. 66(3), pp. 118-127, September 2008.

[8] X. Li, W. T. Liu, Q. Q. Zhi, and W. Zhao, "Three-dimensional joint interpretation of nuclear magnetic resonance and transient electromagnetic data," Chinese Journal of Geophysics-Chinese Edition, vol. 58(8), pp. 2730-2744, August 2015.

[9] J. O. Walbrecker, M. Hertrich, and A. G. Green, "Off-resonance effects in surface nuclear magnetic resonance," Geophysics, vol. 76(2), pp. G1G12, March 2011

[10] D. V. Trushkin, O. A. Shushakov, and A. V.Legchenko, "Modulation effects in non-drilling NMR in the Earth's field," Applied Magnetic Resonance, vol. 5(3-4), pp. 399-406, September 1993.

[11] D. Grombacher, and R. Knight, "The impact of off-resonance effects on water content estimates in surface nuclear magnetic resonance," Geophysics, vol. 80(6), pp. E329-E342, August 2015.

[12] P. B. Weichman, E. M. Lavely, and M. H. Ritzwoller, "Theory of surface nuclear magnetic resonance with applications to geophysical imaging problems," Physical Review E, vol. 62(1), pp. 1290, July 2000. 